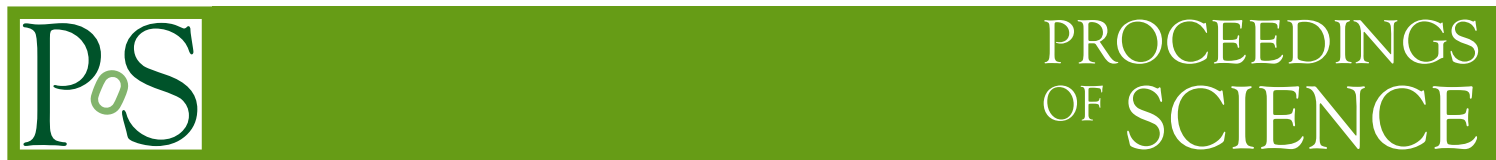

\title{
Chiral effective field theory on the lattice: $A b$ initio calculations of nuclei
}

\author{
Dean Lee ${ }^{* \dagger}$ \\ Department of Physics, \\ North Carolina State University, \\ Box 8202, Raleigh, NC 27695, USA \\ E-mail: dean_lee@ncsu.edu
}

\begin{abstract}
This proceedings article reviews recent results by the Nuclear Lattice EFT Collaboration on an excited state of the ${ }^{12} \mathrm{C}$ nucleus known as the Hoyle state. The Hoyle state plays a key role in the production of carbon via fusion of three alpha particles in red giant stars. Our collaboration has completed lattice calculations which show the structure of the Hoyle state, along with evidence for a low-lying spin-2 rotational excitation. For the ${ }^{12} \mathrm{C}$ ground state and the first excited spin-2 state, we find a compact triangular configuration of alpha clusters. For the Hoyle state and the second excited spin-2 state, we find a bent-arm or obtuse triangular configuration of alpha clusters.
\end{abstract}

The 7th International Workshop on Chiral Dynamics, August 6 -10, 2012

Jefferson Lab, Newport News, Virginia, USA

\footnotetext{
${ }^{*}$ Speaker.

${ }^{\dagger}$ For the Nuclear Lattice EFT Collaboration
} 
Carbon-12 is produced by fusion of three alpha particles in red giant stars. Without some type of enhancement, however, the triple alpha reaction is too slow to account for the observed abundance of carbon in the Universe. Öpik and Salpeter noted that the first step of merging two alpha particles is enhanced by the formation of ${ }^{8} \mathrm{Be}[1,2,3]$. However, a year later Hoyle realized that this enhancement is still insufficient. To resolve this discrepancy, Hoyle predicted an unobserved positive-parity resonance of ${ }^{12} \mathrm{C}$ just above the combined masses of ${ }^{8} \mathrm{Be}$ and ${ }^{4} \mathrm{He}$ [4].

Soon after, Cook, Fowler, Lauritsen and Lauritsen observed a $J^{\pi}=0^{+}$state $278 \mathrm{keV}$ above the ${ }^{8} \mathrm{Be}-{ }^{4} \mathrm{He}$ threshold [5]. This excited $0_{2}^{+}$state is now commonly known as the "Hoyle state". The triple alpha reaction is completed when the Hoyle state decays electromagnetically to the $2_{1}^{+}$ state and subsequently to the $0_{1}^{+}$ground state. Around the same time, Morinaga conjectured that the structure of excited alpha-nuclei such as the Hoyle state may be non-spherical [6], which would imply low-lying rotational excitations of even parity. Other ideas also exist for the structure of the Hoyle state, such as a diffuse trimer of alpha particles [7]. Recently, the spin-2 excitation of the Hoyle state has attracted interest from several experimental groups $[8,9,10,11,12]$.

Our collaboration had published an ab initio lattice calculation of the Hoyle state [13] where the low-lying spectrum of ${ }^{12} \mathrm{C}$ was explored using the framework of chiral effective field theory and Monte Carlo lattice calculations. However the central question regarding the alpha cluster structure of the Hoyle state remained unsolved. In [14] we published new lattice results that answer questions about the structure of the Hoyle state and the existence of rotational excitations. We find evidence for a low-lying spin-2 rotational excitation of the Hoyle state. For the Hoyle state and its spin-2 excitation, we find strong overlap with a "bent-arm" or obtuse triangular configuration of alpha clusters. This is in contrast with the ${ }^{12} \mathrm{C}$ ground state and the first spin- 2 state, where we note strong overlap with a compact triangular configuration of alpha clusters.

Chiral effective field theory handles the interactions of protons and neutrons as a systematic expansion in powers of nucleon momenta and the pion mass. A recent review can be found in Ref. [19]. The low-energy expansion is organized in powers of $Q$, where $Q$ denotes the typical particle momentum and is taken to be the same size as the mass of the pion. The most important contributions to the nuclear Hamiltonian appear at leading order (LO) or $\mathscr{O}\left(Q^{0}\right)$, while the nextto-leading order (NLO) terms are $\mathscr{O}\left(Q^{2}\right)$. In the lattice calculations presented here, we include all possible interactions up to next-to-next-to-leading order (NNLO), or $\mathscr{O}\left(Q^{3}\right)$.

Our analysis uses a periodic cubic lattice with a lattice spacing of $a=1.97 \mathrm{fm}$ and a length of $L=12 \mathrm{fm}$. In the time direction, we use a step size of $a_{t}=1.32 \mathrm{fm}$ and vary the propagation time $L_{t}$ to extrapolate to the limit $L_{t} \rightarrow \infty$. The nucleons are treated as point-like particles on lattice sites, and interactions due to the exchange of pions and multi-nucleon operators are generated using auxiliary fields. Lattice effective field theory was originally used to calculate the manybody properties of homogeneous nuclear and neutron matter [20,21]. Since then, the properties of several atomic nuclei have been investigated $[22,23]$. A recent review of the literature can be found in Ref. [24].

Euclidean time propagation is used to project on to low-energy states of the interacting system. Let $H$ be the Hamiltonian. For any initial quantum state $\Psi$, the projection amplitude is defined as the expectation value $\left\langle e^{-H t}\right\rangle_{\Psi}$. For large Euclidean time $t$, the exponential operator $e^{-H t}$ enhances the signal of low-energy states. Energies can then be determined from the exponential decay of these amplitudes. The first and last few time steps are evaluated using a simpler Hamiltonian 
(I)

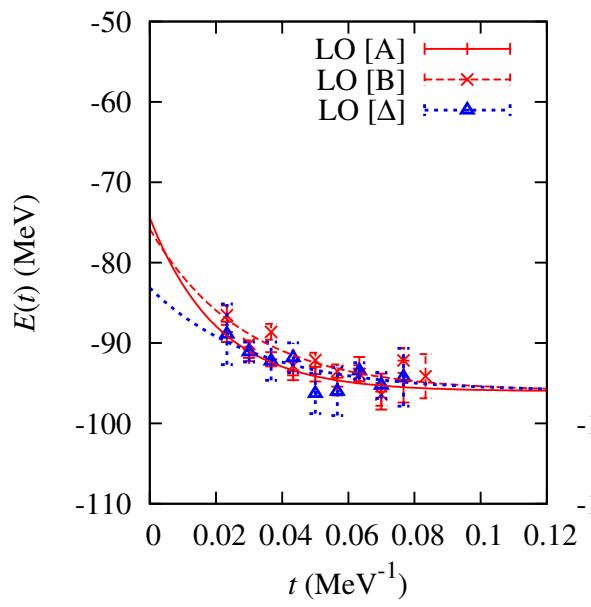

(II)

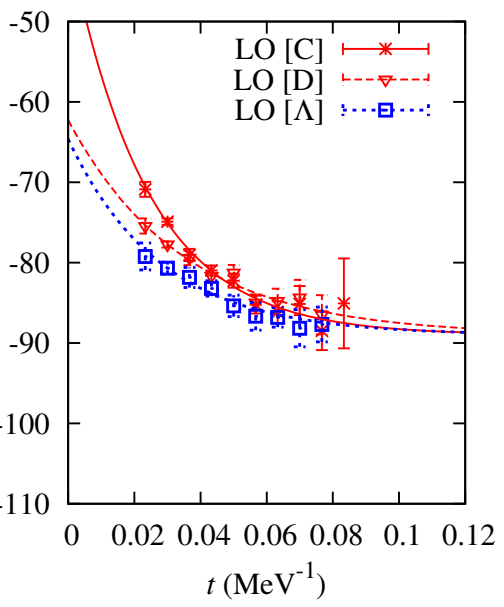

Figure 1: Lattice results for the ${ }^{12} \mathrm{C}$ spectrum at leading order (LO). Panel I shows the results using initial states $A, B$ and $\Delta$, each of which approaches the ground state energy. Panel II shows the results using initial states $C, D$ and $\Lambda$. These trace out an intermediate plateau at an energy $\simeq 7 \mathrm{MeV}$ above the ground state.

$H_{\mathrm{SU}(4)}$, based upon the Wigner SU(4) symmetry for protons and neutrons [25]. Such a Hamiltonian is computationally inexpensive and is used as a low-energy filter before the main calculation. This technique is described in Ref. [24].

We use many different initial and final states in order to probe the structure of the various ${ }^{12} \mathrm{C}$ states. For the ${ }^{12} \mathrm{C}$ states investigated here, we measure four-nucleon correlations by calculating the expectation value $\left\langle\rho^{4}\right\rangle$, where $\rho$ is the total nucleon density. We find strong four-nucleon correlations consistent with the formation of alpha clusters. In Fig. 1, we present lattice results for the energy of ${ }^{12} \mathrm{C}$ at leading order versus Euclidean projection time $t$. For each of the initial states $A-D$, we start with delocalized nucleon standing waves and use a strong attractive interaction in $H_{\mathrm{SU}(4)}$ to allow the nucleons to self-organize into a nucleus. For the initial states $\Delta$ and $\Lambda$, we use alpha cluster wave functions to recover the same states found using the initial states $A-D$. For these calculations, the interaction in $H_{\mathrm{SU}(4)}$ is not as strong and the projected states retain their original alpha cluster character.

In Panel I of Fig. 1, we show lattice results corresponding to the initial states $A, B$, and $\Delta$, each approaching the ground state energy $-96(2) \mathrm{MeV}$. For initial state $A$, we start with four nucleons (each at zero momentum) apply creation operators after the first time step to inject four more nucleons at rest, followed by the injection of four additional nucleons at rest after the second time step. This procedure is used in reverse to extract nucleons for the final state $A$. An identical scheme is used for initial state $B$, though the interactions in $H_{\mathrm{SU}(4)}$ are not as strongly attractive as those for $A$.

For the initial state $\Delta$, we use a wave function constructed from of three alpha clusters, as shown in Fig. 2. The alpha clusters are formed by Gaussian wave packets centered on the vertices of a compact triangle. In order to construct eigenstates of total momentum and lattice cubic rotations, we consider all possible translations and rotations of the initial state. There are a total of 12 
equivalent orientations of $\Delta$. We do not find rapid convergence to the ground state when starting from any other configuration of alpha clusters. We thus conclude that the alpha cluster configurations in Fig. 2 have the strongest overlap with the $0_{1}^{+}$ground state of ${ }^{12} \mathrm{C}$. The fact that $\Delta$ is an isosceles right triangle rather than an equilateral triangle is merely a lattice artifact.

In Panel II of Fig. 1, we show the leading-order energies obtained from initial states $C, D$, and $\Lambda$. Each of these approaches an intermediate plateau at $-89(2) \mathrm{MeV}$. In the limit of infinite Euclidean time, these would eventually also approach the ground state energy. However, it is clear that a different state is first being formed that is distinct from the ground state. We identify the $0^{+}$ state in this plateau region as the $0_{2}^{+}$Hoyle state. The common thread connecting the initial states $C, D$, and $\Lambda$ is that each produces a state which has an extended (or prolate) geometry. This is in contrast to the oblate triangular configuration shown in Fig. 2.

For initial state $C$, we start with four nucleons at rest, four with momenta $(2 \pi / L, 2 \pi / L, 2 \pi / L)$, and four with momenta $(-2 \pi / L,-2 \pi / L,-2 \pi / L)$. Similarly, for initial state $D$ we take four nucleons at rest, four with momenta $(2 \pi / L, 2 \pi / L, 0)$, and four with momenta $(-2 \pi / L,-2 \pi / L, 0)$. Finally, initial state $\Lambda$ uses a set of three alpha clusters formed by Gaussian wave packets centered on the vertices of a "bent-arm" (or obtuse) triangular configuration, as shown in Fig. 3. There are a total of 24 equivalent orientations of $\Lambda$. We do not find the same energy plateau starting from other configurations of alpha clusters. We conclude that configurations of the type shown in Fig. 3 have the strongest overlap with the $0_{2}^{+}$Hoyle state of ${ }^{12} \mathrm{C}$.

We use the multi-channel method of Ref. [13] to find a spin-2 excitation above the ground state, as well as a spin-2 excitation above the Hoyle state. In both cases, we make use of the $E^{+}$ representation of the cubic rotation group on the lattice. We summarize our results for the binding energies of the low-lying, even-parity states of ${ }^{12} \mathrm{C}$ in Table 1 . The binding energies at NNLO are in agreement with the experimental values.

\section{Acknowledgments}

The author thankful for discussions with the other members of the Nuclear Lattice EFT Collaboration, Evgeny Epelbaum, Hermann Krebs, Timo A. Lähde, Ulf-G. Meißner, and Gautam

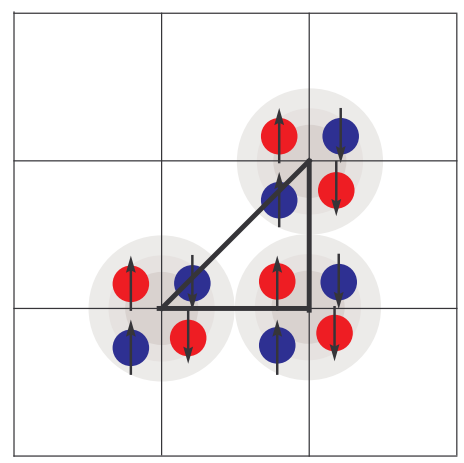

Figure 2: Illustration of the initial state $\Delta$. There are 12 equivalent orientations of this compact triangular configuration. 


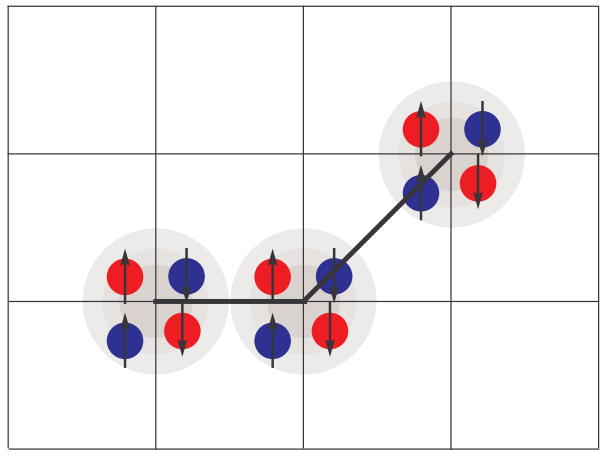

Figure 3: Illustration of the initial state $\Lambda$. There are 24 equivalent orientations of this "bent-arm" or obtuse triangular configuration.

Table 1: Lattice and experimental results for the energies of the low-lying even-parity states of ${ }^{12} \mathrm{C}$, in units of $\mathrm{MeV}$.

\begin{tabular}{|c||c|c|c|c|}
\hline & $0_{1}^{+}$ & $2_{1}^{+}\left(E^{+}\right)$ & $0_{2}^{+}$ & $2_{2}^{+}\left(E^{+}\right)$ \\
\hline LO & $-96(2)$ & $-94(2)$ & $-89(2)$ & $-88(2)$ \\
NLO & $-77(3)$ & $-74(3)$ & $-72(3)$ & $-70(3)$ \\
NNLO & $-92(3)$ & $-89(3)$ & $-85(3)$ & $-83(3)$ \\
\hline & & & & $-82.6(1)[8,10]$ \\
Exp & -92.16 & -87.72 & -84.51 & $-81.1(3)[9]$ \\
& & & & $-82.32(6)[11]$ \\
\hline
\end{tabular}

Rupak. Partial financial support from the DFG and NSFC (CRC 110), HGF (VH-VI-417), BMBF (06BN7008) USDOE (DE-FG02-03ER41260), EU HadronPhysics3 project "Study of strongly interacting matter", and ERC project 259218 NUCLEAREFT. Computational resources were provided by the Jülich Supercomputing Centre (JSC) at the Forschungszentrum Jülich.

\section{References}

[1] E. J. Öpik, Proc. Roy. Irish Acad. A54, 49 (1951).

[2] E. E. Salpeter, Astrophys. J. 115, 326 (1952).

[3] E. E. Salpeter, Ann. Rev. Nucl. Sci. 2, 41 (1953).

[4] F. Hoyle, Astrophys. J. Suppl. 1, 121 (1954).

[5] C. Cook, W. A. Fowler, C. C. Lauritsen, and T. Lauritsen, Phys. Rev. 107, 508 (1957).

[6] H. Morinaga, Phys. Rev. 101, 254 (1956).

[7] A. Tohsaki, H. Horiuchi, P. Schuck, and G. Röpke, Phys. Rev. Lett. 87, 192501 (2001), nucl-th/0110014.

[8] M. Freer et al., Phys. Rev. C 80, 041303 (2009).

[9] S. Hyldegaard et al., Phys. Rev. C 81, 024303 (2010). 
[10] W. R. Zimmerman, N. E. Destefano, M. Freer, M. Gai, and F. D. Smit, Phys. Rev. C 84, 027304 (2011).

[11] M. Itoh et al., Phys. Rev. C 84, 054308 (2011).

[12] F. D. Smit, F. Nemulodi, Z. Buthelezi, J. Carter, R. W. Fearick, S. V. Förtsch, M. Freer, H. Fujita, M. Jingo, C. O. Kureba, et al., Phys. Rev. C 86, 037301 (2012), URL http://link.aps.org/doi/10.1103/PhysRevC.86.037301.

[13] E. Epelbaum, H. Krebs, D. Lee, and U.-G. Meißner, Phys. Rev. Lett. 106, 192501 (2011), arXiv:1101.2547 [nucl-th].

[14] E. Epelbaum, H. Krebs, T. A. Lähde, D. Lee, and U.-G. Meißner, Phys. Rev. Lett. 109, 252501 (2012), arXiv:1208.1328 [nucl-th].

[15] R. Roth, J. Langhammer, A. Calci, S. Binder, and P. Navratil, Phys.Rev.Lett. 107, 072501 (2011), 1105.3173.

[16] C. Forssen, R. Roth, and P. Navratil (2011), 1110.0634.

[17] M. Chernykh, H. Feldmeier, T. Neff, P. von Neumann-Cosel, and A. Richter, Phys. Rev. Lett. 98, 032501 (2007).

[18] M. Chernykh, H. Feldmeier, T. Neff, P. von Neumann-Cosel, and A. Richter, Phys. Rev. Lett. 105, 022501 (2010), 1004.3877.

[19] E. Epelbaum, H.-W. Hammer, and U.-G. Meißner, Rev. Mod. Phys. 81, 1773 (2009a), arXiv:0811.1338 [nucl-th].

[20] H. M. Müller, S. E. Koonin, R. Seki, and U. van Kolck, Phys. Rev. C61, 044320 (2000), nucl-th/9910038.

[21] D. Lee, B. Borasoy, and T. Schäfer, Phys. Rev. C70, 014007 (2004), nucl-th / 0402072.

[22] E. Epelbaum, H. Krebs, D. Lee, and U. G. Meißner, Eur. Phys. J. A41, 125 (2009b), arXiv:0903.1666 [nucl-th].

[23] E. Epelbaum, H. Krebs, D. Lee, and U.-G. Meißner, Phys. Rev. Lett. 104, 142501 (2010a), arXiv:0912.4195 [nucl-th].

[24] D. Lee, Prog. Part. Nucl. Phys. 63, 117 (2009), arXiv: 0804.3501 [nucl-th].

[25] E. Wigner, Phys. Rev. 51, 106 (1937). 